AperTO - Archivio Istituzionale Open Access dell'Università di Torino

\title{
Practical numbers in Lucas sequences
}

\section{This is the author's manuscript}

Original Citation:

\section{Availability:}

This version is available http://hdl.handle.net/2318/1676275

since 2018-09-10T11:24:37Z

Published version:

DOI:10.2989/16073606.2018.1502697

Terms of use:

Open Access

Anyone can freely access the full text of works made available as "Open Access". Works made available under a Creative Commons license can be used according to the terms and conditions of said license. Use of all other works requires consent of the right holder (author or publisher) if not exempted from copyright protection by the applicable law. 


\title{
PRACTICAL NUMBERS IN LUCAS SEQUENCES
}

\author{
CARLO SANNA
}

\begin{abstract}
A practical number is a positive integer $n$ such that all the positive integers $m \leq n$ can be written as a sum of distinct divisors of $n$. Let $\left(u_{n}\right)_{n \geq 0}$ be the Lucas sequence satisfying $u_{0}=0, u_{1}=1$, and $u_{n+2}=a u_{n+1}+b u_{n}$ for all integers $n \geq 0$, where $a$ and $b$ are fixed nonzero integers. Assume $a(b+1)$ even and $a^{2}+4 b>0$. Also, let $\mathcal{A}$ be the set of all positive integers $n$ such that $\left|u_{n}\right|$ is a practical number. Melfi proved that $\mathcal{A}$ is infinite. We improve this result by showing that $\# \mathcal{A}(x) \gg x / \log x$ for all $x \geq 2$, where the implied constant depends on $a$ and $b$. We also pose some open questions regarding $\mathcal{A}$.
\end{abstract}

\section{INTRODUCTION}

A practical number is a positive integer $n$ such that all the positive integers $m \leq n$ can be written as a sum of distinct divisors of $n$. The term "practical" was coined by Srinivasan [7]. Let $\mathcal{P}$ be the set of practical numbers. Estimates for the counting function $\# \mathcal{P}(x)$ were given by Hausman and Shapiro [1], Tenenbaum [10], Margenstern [2], Saias [5], and, finally, Weingartner [12], who proved that there exists a constant $C>0$ such that

$$
\# \mathcal{P}(x)=\frac{C x}{\log x} \cdot\left(1+O\left(\frac{\log \log x}{\log x}\right)\right)
$$

for all $x \geq 3$, settling a conjecture of Margenstern [2].

In analogy with well-known conjectures about prime numbers, Melfi [4] proved that every positive even integer is the sum of two practical numbers, and that there are infinitely many triples $(n, n+2, n+4)$ of practical numbers. Let $\left(u_{n}\right)_{n \geq 0}$ be a Lucas sequence, that is, a sequence of integers satisfying $u_{0}=0, u_{1}=1$, and $u_{n+2}=a u_{n+1}+b u_{n}$ for all integers $n \geq 0$, where $a$ and $b$ are two fixed nonzero integers. Also, let $\mathcal{A}$ be the set of all positive integers $n$ such that $\left|u_{n}\right|$ is a practical number. From now on, we assume $a^{2}+4 b>0$ and $a(b+1)$ even. We remark that, in the study of $\mathcal{A}$, assuming $a(b+1)$ even is not a loss of generality. Indeed, if $a(b+1)$ is odd then $u_{n}$ is odd for all $n \geq 1$ and, since 1 is the only odd practical number, it follows that $\mathcal{A}=\{1\}$. Melfi [3, Theorem 10] proved the following result.

Theorem 1.1. The set $\mathcal{A}$ is infinite. Precisely, $2^{j} \cdot 3 \in \mathcal{A}$ for all sufficiently large positive integers $j$, how large depending on $a$ and $b$, and hence

$$
\# \mathcal{A}(x) \gg \log x
$$

for all sufficiently large $x>1$.

In this paper, we improve Theorem 1.1 to the following:

Theorem 1.2. For all $x \geq 2$, we have

$$
\# \mathcal{A}(x) \gg \frac{x}{\log x},
$$

where the implied constant depends on $a$ and $b$.

We leave the following open questions to the interested readers:

(Q1) Does $\mathcal{A}$ have zero natural density?

(Q2) Can a nontrivial upper bound for $\# \mathcal{A}(x)$ be proved?

2010 Mathematics Subject Classification. Primary: 11B37, Secondary: 11B39, 11N25.

Key words and phrases. Fibonacci numbers; Lucas sequences; practical numbers. 
(Q3) Are there infinitely many nonpractical $n$ such that $\left|u_{n}\right|$ is practical?

(Q4) Are there infinitely many practical $n$ such that $\left|u_{n}\right|$ is nonpractical?

(Q5) What about practical numbers in general integral linear recurrences over the integers?

Notation. For any set of positive integers $\mathcal{S}$, we put $\mathcal{S}(x):=\mathcal{S} \cap[1, x]$ for all $x \geq 1$, and \# $\mathcal{S}(x)$ denotes the counting function of $\mathcal{S}$. We employ the Landau-Bachmann "Big Oh" notation $O$, as well as the associated Vinogradov symbols $\ll$ and $\gg$, with their usual meanings. Any dependence of the implied constants is explicitly stated. As usual, we write $\mu(n), \varphi(n), \sigma(n)$, and $\omega(n)$, for the Möbius function, the Euler's totient function, the sum of divisors, and the number of prime factors of a positive integer $n$, respectively.

\section{Preliminaries on LuCAS SEquences}

In this section we collect some basic facts about Lucas sequences. Let $\alpha$ and $\beta$ be the two roots of the characteristic polynomial $X^{2}-a X-b$. Since $a^{2}+4 b>0$ and $b \neq 0$, we have that $\alpha$ and $\beta$ are real, nonzero, and distinct. It is well known that the generalized Binet's formula

$$
u_{n}=\frac{\alpha^{n}-\beta^{n}}{\alpha-\beta}
$$

holds for all integers $n \geq 0$. Define

$$
\Phi_{n}:=\prod_{\substack{1 \leq k \leq n \\ \operatorname{gcd}(n, k)=1}}\left(\alpha-e^{2 \pi \mathrm{i} k / n} \beta\right)
$$

for each positive integer $n$. It can be proved that $\Phi_{n} \in \mathbb{Z}$ for all integers $n>1$ (see, e.g., [9, p. 428]). Furthermore, we have

$$
u_{n}=\prod_{\substack{d \mid n \\ d>1}} \Phi_{d}
$$

and, by the Möbius inversion formula,

$$
\Phi_{n}=\prod_{d \mid n} u_{n / d}^{\mu(d)}
$$

for all integers $n>1$. Changing the sign of $a$ changes the signs of $\alpha, \beta$ and turns $u_{n}$ into $(-1)^{n+1} u_{n}$, which is not a problem, since for the study of $\mathcal{A}$ we are interested only in $\left|u_{n}\right|$. Hence, without loss of generality, we can assume $a>0$ and $\alpha>|\beta|$, which in turn implies that $u_{n}, \Phi_{n}>0$ for all integers $n>0$. We conclude this section with an easy lemma regarding the growth of $u_{n}$ and $\Phi_{n}$.

Lemma 2.1. For all integers $n>0$, we have

(i) $u_{n} \geq u_{n-1}$;

(ii) $u_{n}=\alpha^{n+O(1)}$;

(iii) $\Phi_{n}=\alpha^{\varphi(n)+O(1)}$;

where the implied constants depend on $a$ and $b$.

Proof. If $b>0$, then (i) is clear from the recursion for $u_{n}$. Hence, suppose $b<0$, so that $\beta>0$. After a bit of manipulations, (i) is equivalent to $\alpha^{n-1}(\alpha-1) \geq \beta^{n-1}(\beta-1)$, which in turn follows easily since $\alpha>\beta>0$. Claim (ii) is a consequence of (1). Setting $\gamma:=\beta / \alpha$, by (1) and (3), we get

$$
\Phi_{n}=\alpha^{\varphi(n)} \prod_{d \mid n}\left(\frac{1-\gamma^{n / d}}{\alpha-\beta}\right)^{\mu(d)}=\alpha^{\varphi(n)} \prod_{d \mid n}\left(1-\gamma^{n / d}\right)^{\mu(d)}
$$


for all integers $n>1$, where we used the well-known formulas $\sum_{d \mid n} \mu(d) \frac{n}{d}=\varphi(n)$ and $\sum_{d \mid n} \mu(d)=0$. Therefore, since $|\gamma|<1$, we have

$$
\left|\log \left(\Phi_{n} / \alpha^{\varphi(n)}\right)\right| \leq \sum_{d \mid n}\left|\log \left(1-\gamma^{d}\right)\right| \ll \sum_{d=1}^{\infty}|\gamma|^{d} \ll 1,
$$

and also (iii) is proved.

\section{Preliminaries on PRACtical nUmbers AND ClOSE Relatives}

The following lemma on practical numbers will be fundamental later.

Lemma 3.1. If $n$ is a practical number and $m \leq 2 n$ is a positive integer, then $m n$ is a practical number.

Proof. See [4, Lemma 1].

Close relatives of practical numbers are $\varphi$-practical numbers. A $\varphi$-practical number is a positive integer $n$ such that all the positive integers $m \leq n$ can be written as $m=\sum_{d \in \mathcal{D}} \varphi(d)$, where $\mathcal{D}$ is a subset of the divisors of $n$. This notion was introduced by Thompson [11] while studying the degrees of the divisors of the polynomial $X^{n}-1$. Indeed, $\varphi$-practical numbers are exactly the positive integers $n$ such that $X^{n}-1$ has a divisor of every degree up to $n$.

We need a couple of results regarding $\varphi$-practical numbers.

Lemma 3.2. Let $n$ be a $\varphi$-practical number and $p$ be a prime number not dividing $n$. Then pn is $\varphi$-practical if and only if $p \leq n+2$. Moreover, $p^{j} n$ is $\varphi$-practical if and only if $p \leq n+1$, for every integer $j \geq 2$.

Proof. See [11, Lemma 4.1].

Lemma 3.3. If $n$ is an even $\varphi$-practical number, and if $d_{1}, \ldots, d_{s}$ are all the divisors of $n$ ordered so that $\varphi\left(d_{1}\right) \leq \cdots \leq \varphi\left(d_{s}\right)$, then

$$
\varphi\left(d_{j+1}\right) \leq \sum_{i=1}^{j} \varphi\left(d_{i}\right)
$$

for all positive integers $j<s$.

Proof. It is not difficult to see that $n$ is $\varphi$-practical if and only if

$$
\varphi\left(d_{j+1}\right) \leq 1+\sum_{i=1}^{j} \varphi\left(d_{i}\right)
$$

for all positive integers $j<s$ (see [11, p. 1041]). Hence, we have only to prove that $n$ even ensures that in (5) the equality cannot happen. If $j=1$ then (4) is obvious since $\left\{d_{1}, d_{2}\right\}=\{1,2\}$, so we can assume $1<j<s$. At this point $\varphi\left(d_{j+1}\right)$ is even, while

$$
1+\sum_{i=1}^{j} \varphi\left(d_{i}\right)
$$

is odd, because $\varphi(m)$ is even for all integers $m>2$. Thus, in (5) the equality is not satisfied.

Let $\theta$ be a real-valued arithmetic function, and define $\mathcal{B}_{\theta}$ as the set containing $n=1$ and all those $n=p_{1}^{a_{1}} \cdots p_{k}^{a_{k}}$, where $p_{1}<\cdots<p_{k}$ are prime numbers and $a_{1}, \ldots, a_{k}$ are positive integers, which satisfy

$$
p_{j} \leq \theta\left(\prod_{i=1}^{j-1} p_{i}^{a_{i}}\right)
$$

for $j=1, \ldots, k$, where the empty product is equal to 1 . If $\theta(n):=\sigma(n)+1$, then $\mathcal{B}_{\theta}$ is the set of practical numbers. This is a characterization given by Stewart [8] and Sierpiński [6]. 
Weingartner proved a general and strong estimate for $\# \mathcal{B}_{\theta}(x)$. The following is a simplified version adapted just for our purposes.

Theorem 3.4. Suppose $\theta(1) \geq 2$ and $n \leq \theta(n) \leq$ An for all positive integers $n$, where $A \geq 1$ is a constant. Then, we have

as $x \rightarrow+\infty$, where $c_{\theta}>0$ is a constant.

$$
\# \mathcal{B}_{\theta}(x) \sim \frac{c_{\theta} x}{\log x}
$$

Proof. See [12, Theorems 1.2 and 5.1].

\section{Proof of Theorem 1.2}

The key tool of the proof is the following technical lemma.

Lemma 4.1. Suppose that $n$ is a sufficiently large positive integer, how large depending on a and $b$. Let $p$ be a prime number and write $n=p^{v} m$ for some nonnegative integer $v$ and some positive integer $m$ not divisible by $p$. If $m$ is an even $\varphi$-practical number, $n \in \mathcal{A}$, and $p<m$, then $p^{k} n \in \mathcal{A}$ for all positive integers $k$.

Proof. Clearly, it is enough to prove the claim for $k=1$. Let $d_{1}=1, d_{2}=2, \ldots, d_{s}$ be all the divisors of $m$, ordered to that $\varphi\left(d_{1}\right) \leq \cdots \leq \varphi\left(d_{s}\right)$. Furthermore, define

$$
N_{j}:=u_{n} \prod_{i=1}^{j} \Phi_{p^{v+1} d_{i}},
$$

for $j=1, \ldots, s$. We shall prove that each $N_{j}$ is practical. This implies the thesis, since $N_{s}=u_{p n}$ by (2).

We proceed by induction on $j$. First, by (2) and Lemma 2.1(i), we have

$$
\Phi_{p^{v+1} d_{1}}=\Phi_{p^{v+1}} \leq u_{p^{v+1}} \leq u_{p^{v} m}=u_{n}
$$

since $p<m$. Hence, applying Lemma 3.1 and the fact that $u_{n}$ is practical, we get that $N_{1}=u_{n} \Phi_{p^{v+1} d_{1}}$ is practical.

Now assuming that $N_{j}$ is practical we shall prove that $N_{j+1}$ is practical. Again, since $N_{j+1}=\Phi_{p^{v+1} d_{j+1}} N_{j}$, thanks to Lemma 3.1 it is enough to show that the inequality

$$
\Phi_{p^{v+1} d_{j+1}} \leq u_{n} \prod_{i=1}^{j} \Phi_{p^{v+1} d_{i}}
$$

holds. In turn, by Lemma 2.1(ii) and (iii), we have that (6) is implied by

$$
n+\varphi\left(p^{v+1}\right)\left[-\varphi\left(d_{j+1}\right)+\sum_{i=1}^{j} \varphi\left(d_{i}\right)\right] \geq C(j+1),
$$

where $C>0$ is a constant depending only on $a$ and $b$.

On the one hand, since $m$ is an even $\varphi$-practical number, by Lemma 3.3 we have that the term of (7) in square brackets is nonnegative. On the other hand, for sufficiently large $n$, we have

$$
n \geq C(\log n / \log 2+1) \geq C(\omega(n)+1) \geq C(j+1) .
$$

Therefore, (7) holds and the proof is complete.

We are ready to prove Theorem 1.2. Pick a sufficiently large positive integer $h$, depending on $a$ and $b$, such that the claim of Lemma 4.1 holds for all integers $n \geq 2^{h} \cdot 3$. Moreover, by Theorem 1.1, we can assume that $2^{j} \cdot 3 \in \mathcal{A}$ for all integers $j \geq h$. Put $\mathcal{B}:=\mathcal{B}_{\theta} \backslash\{1\}$, where $\theta(n):=\max \{2, n\}$. Note that, as a consequence of Lemma 3.2, all the elements of $\mathcal{B}$ are even $\varphi$-practical numbers. We shall prove that for all $n \in \mathcal{B}$ we have $2^{h} \cdot 3 n \in \mathcal{A}$. In this way, thanks to Theorem 3.4, we get

$$
\# \mathcal{A}(x) \geq \# \mathcal{B}\left(\frac{x}{2^{h} \cdot 3}\right) \gg \frac{x}{\log x}
$$


for all sufficiently large $x$. Hence, since $1 \in \mathcal{A}$, Theorem 1.2 follows.

We proceed by induction on the number of prime factors of $n \in \mathcal{B}$. If $n \in \mathcal{B}$ has exactly one prime factor, then it follows easily that $n=2^{j}$ for some positive integer $j$. Hence, we have $2^{h} \cdot 3 n=2^{h+j} \cdot 3 \in \mathcal{A}$, as claimed.

Now, assuming that the claim is true for all $n \in \mathcal{B}$ with exactly $k \geq 1$ prime factors, we shall prove it for all $n \in \mathcal{B}$ having $k+1$ prime factors. Write $n=p_{1}^{a_{1}} \cdots p_{k+1}^{a_{k+1}}$, where $p_{1}<\cdots<p_{k+1}$ are prime numbers and $a_{1}, \ldots, a_{k+1}$ are positive integers. Put also $m:=p_{1}^{a_{1}} \cdots p_{k}^{a_{k}}$. Since $n \in \mathcal{B}$, we have $m \in \mathcal{B}$ and $p_{k+1}<m$. On the one hand, by the induction hypothesis, $2^{h} \cdot 3 m \in \mathcal{A}$. On the other hand, it is easy to see that $m \in \mathcal{B}$ implies $2^{h} m \in \mathcal{B}$ and $2^{h} \cdot 3 m \in \mathcal{B}$.

First, suppose $p_{k+1}>3$. Since $2^{h} \cdot 3 m$ is an even $\varphi$-practical number, $2^{h} \cdot 3 m \in \mathcal{A}$, and $p_{k+1}<2^{h} \cdot 3 m$, by Lemma 4.1 we get that $2^{h} \cdot 3 n=2^{h} \cdot 3 m p_{k+1}^{a_{k+1}} \in \mathcal{A}$, as claimed.

On the other hand, if $p_{k+1}=3$ the situation is similar. Since $2^{h} m$ is an even $\varphi$-practical number, $2^{h} \cdot 3 m \in \mathcal{A}$, and $p_{k+1}<2^{h} m$, by Lemma 4.1 we get that $2^{h} \cdot 3 n=2^{h} \cdot 3 m p_{k+1}^{a_{k+1}} \in \mathcal{A}$, as claimed. The proof is complete.

Acknowledgements. The authors thanks the anonymous referee for carefully reading the paper. The author is a member of the INdAM group GNSAGA.

\section{REFERENCES}

1. M. Hausman and H. N. Shapiro, On practical numbers, Comm. Pure Appl. Math. 37 (1984), no. 5, 705-713.

2. M. Margenstern, Les nombres pratiques: théorie, observations et conjectures, J. Number Theory 37 (1991), no. $1,1-36$.

3. G. Melfi, A survey on practical numbers, Rend. Sem. Mat. Univ. Politec. Torino 53 (1995), no. 4, 347-359, Number theory, II (Rome, 1995).

4. G. Melfi, On two conjectures about practical numbers, J. Number Theory 56 (1996), no. 1, 205-210.

5. E. Saias, Entiers à diviseurs denses. I, J. Number Theory 62 (1997), no. 1, 163-191.

6. W. Sierpiński, Sur une propriété des nombres naturels, Ann. Mat. Pura Appl. (4) 39 (1955), 69-74.

7. A. K. Srinivasan, Practical numbers, Current Sci. 17 (1948), 179-180. MR 0027799

8. B. M. Stewart, Sums of distinct divisors, Amer. J. Math. 76 (1954), 779-785.

9. C. L. Stewart, On divisors of Fermat, Fibonacci, Lucas, and Lehmer numbers, Proc. London Math. Soc. (3) 35 (1977), no. 3, 425-447.

10. G. Tenenbaum, Sur un problème de crible et ses applications, Ann. Sci. École Norm. Sup. (4) 19 (1986), no. $1,1-30$.

11. L. Thompson, Polynomials with divisors of every degree, J. Number Theory 132 (2012), no. 5, $1038-1053$.

12. A. Weingartner, Practical numbers and the distribution of divisors, Q. J. Math. 66 (2015), no. 2, 743-758.

Università degli Studi di Torino, Department of Mathematics, Turin, Italy

E-mail address: carlo.sanna.dev@gmail.com 